\title{
Performance of 4- and 8-State TCM Schemes with Asymmetric 8-PSK in Fading Channels
}

\author{
L. Venkata Subramaniam, Member, IEEE, B. Sundar Rajan, Senior Member, IEEE, and Rajendar Bahl, Member, IEEE
}

\begin{abstract}
For Rayleigh fading channels, Ricean fading channels with a small parameter and shadowed Rician channels with the code design criteria are to maximize the effective length as well as the minimum product distance of the code. In this paper, we use two different asymmetric 8-PSK signal sets in 4-state and 8-state rate $2 / 3$ trellis-coded modulation (TCM) schemes and show performance gain over TCM schemes with symmetric 8-PSK constellations. The performance gain is due to an increase in the minimum product distance compared to that of the best known TCM schemes of comparable states using symmetric 8-PSK signal sets while the effective length remains same. Simulation is performed over the Rayleigh, Ricean, and shadowed Rician fading channels to demonstrate the performance gain of the asymmetric 8-PSK TCM schemes over the symmetric 8-PSK TCM.
\end{abstract}

Index Terms-Asymmetric phase shift keying, fading channels, trellis-coded modulation.

\section{INTRODUCTION}

$\mathbf{T}$ RELLIS-CODED modulation (TCM) schemes achieve good power and bandwidth efficiency for additive white Gaussian noise (AWGN) channels [1]. In recent years, TCM has increasingly been used in such channels as the mobile radio and indoor wireless channels, which are often modeled as a fading channel [2]-[8]. Recently, this channel is also being used for data transmission, in addition to speech. Typically, the acceptable speech error rate is $10^{-3}$, while data error rates smaller than $10^{-5}$ are necessary. In this paper, we consider combining TCM schemes with asymmetric PSK (APSK) signal sets as a means of improving the minimum product distance and improving the performance especially at data error rates. Asymmetry has been introduced in the 8-PSK signal set in such a way as to improve the minimum product distance. The type of asymmetry in the 4-state and 8-state cases differ.

The code design criteria for Rayleigh fading channels specifies that the effective length and the minimum product distance should be maximized for the code and the multiciplicity should

Manuscript received August 28, 1998; revised February 23, 1999. The work of B. S. Rajan was supported in part by CSIR, India, under research grant 25(0086)/97/EMR-II. Part of this paper was presented at the 1998 International Symposium on Information Theory held at the Massachusetts Institute of Technology, Cambridge, MA, on August 16-21, 1998.

L. V. Subramaniam was with the Centre for Applied Research in Electronics, Indian Institute of Technology, Delhi, India. He is now with the IBM Solutions Research Centre, Indian Institute of Technology, New Delhi 110 016, India (e-mail: 1vsubram@in.ibm.com).

B. S. Rajan is with the Department of Electrical Communication Engineering, Indian Institute of Science, Bangalore 560 012, India (e-mail bsrajan@ece.iisc.ernet.in).

R. Bahl is with the Centre for Applied Research in Electronics, Indian Institute of Technology, New Delhi 110 016, India (e-mail: rbahl@ care.iitd.ernet.in).

Publisher Item Identifier S 0018-9545(00)00919-1. be minimized [3]. Four-state rate $2 / 3$ symmetric 8 -PSK TCM schemes have been studied in connection with use in Rayleigh and shadowed mobile radio channels [2], [7], [8]. Fig. 1(a) shows the symmetric 8-PSK signal set and Fig. 1(b) and (c) shows the asymmetric 8-PSK signal sets used in this paper instead of symmetric 8-PSK signal set. Since the presence of parallel transitions in the trellis associated with the TCM scheme limits the effective length to one, it should be avoided for applications in fading channels. Fig. 2(a) shows the state transitions of a fully connected, 4-state TCM scheme without parallel transitions with signal points associated with transitions given by Fig. 2(b) for Wilson and Leung code [7] and Fig. 2(c) for Jamali and LeNgoc code [8]. Wilson and Leung have suggested a scheme that results in an effective length of two, which is the maximum achievable time diversity with 4-state 8-PSK TCM schemes. However, the minimum product distance has not been optimized resulting in a path with actual length 3 and effective length 2 with product distance of $1.172 E_{s}^{2}$, where $\sqrt{E_{s}}$ is the radius of the circle in Fig. 1(a) [2, ch. 5]. Jamali and LeNgoc describe design rules which optimize the minimum product distance and improve the same to $2.344 E_{s}^{2}$ while maintaining the effective length at 2 resulting in signal point assignment as shown in Fig. 2(c). In this paper, using the signal point assignments of [8] we further improve the performance using an asymmetric 8-PSK signal set shown in Fig. 1(b). Du and Vucetic [9] match the performance of Jamali and LeNgoc code with Gray code labeling of the 8-PSK signal set.

The 8-state rate 2/3 8-PSK Ungerboeck code [1] has a trellis without parallel transitions. Fig. 3 shows the state transitions of the 8-state Ungerboeck code. It achieves the maximum effective length of two that is possible for an 8 -state rate $2 / 38$-PSK code. This code has a minimum product distance of $8 E_{s}^{2}$. This is the best known 8 -state rate 2/3 8-PSK TCM scheme for fading channels (incidentally also for AWGN channels). The use of trellis coding with asymmetric modulations has been considered earlier by Divsalar, Simon, and Yuen, in [10], for AWGN and Divsalar and Simon in [6] for fading channels. In [10] and [6], the asymmetric M-PSK signal set is created by adding together the symmetrical M/2-PSK signal set with a rotated version of itself and the signal point assignment was made by "mapping by set partitioning" [1]. The asymmetric 8-PSK constellation used by Divsalar et al. in [10] and [6] is shown in Fig. 1(b). The angle of rotation is optimized for the channel. However, for 4-state and 8-state rate $2 / 3$ 8-PSK schemes the use of asymmetry in this way has not given any improvement over the symmetric coded signal set. We show that introducing asymmetry as shown in Fig. 1(c) leads to performance improvement for the 8-state rate 2/3 8-PSK Ungerboeck code. Notice that the type of asymmetry 


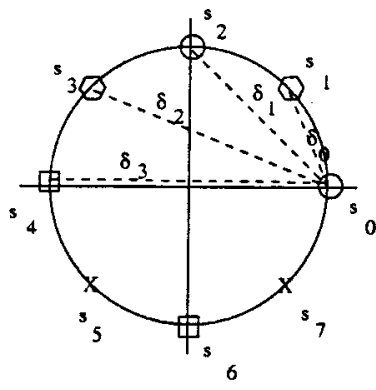

(a)

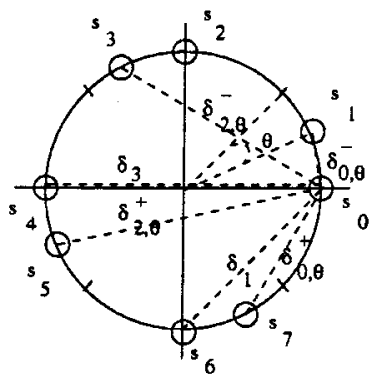

(b)

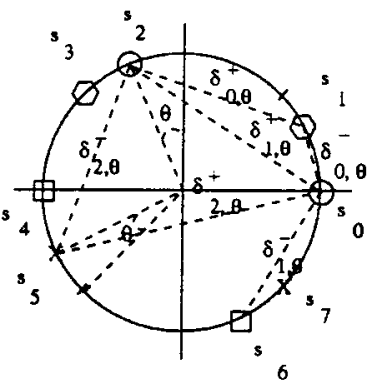

(c)

Fig. 1. (a) Symmetric 8-PSK with distances marked. (b) Asymmetric 8-PSK (distances marked used in Section III-A). (c) Asymmetric 8-PSK (distances marked as well as the pairings of signal points shown by similar shapes are used in Section III-B).

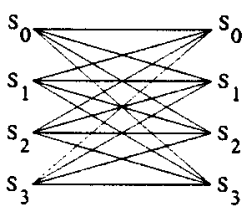

(a)

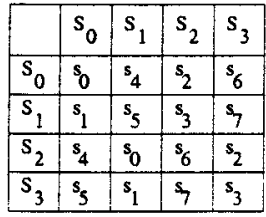

(b)

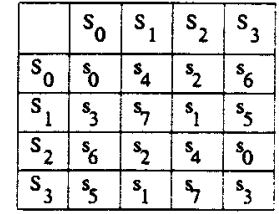

(c)
Fig. 2. (a) State transition diagram of a fully connected 4-state TCM scheme. (b) Wilson and Leung. (c) Jamali and LeNgoc.

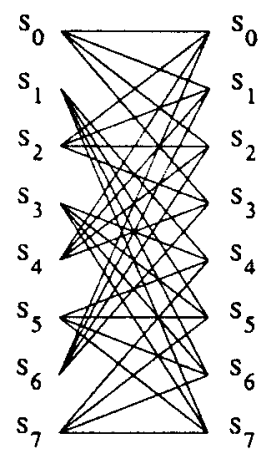

(a)

\begin{tabular}{|c|c|c|c|c|c|c|c|c|}
\hline & $s_{0}$ & $s_{1}$ & $s_{2}$ & $\mathrm{~s}_{3}$ & $\mathrm{~S}_{4}$ & $S_{5}$ & $S_{6}$ & $\mathrm{~s}_{7}$ \\
\hline$s_{0}$ & $s_{0}$ & $s_{4}$ & $s_{2}$ & ${ }_{6} 6$ & $x$ & $x$ & $x$ & \\
\hline$s_{1}$ & $x$ & $\mathbf{x}$ & $x$ & $x$ & $s_{1}$ & $s_{5}$ & $\mathrm{~s}_{3}$ & $s_{7}$ \\
\hline$s_{2}$ & $s_{4}$ & $s_{0}$ & ${ }^{s} 6$ & $s_{2}$ & $x$ & $\mathbf{x}$ & $x$ & \\
\hline $\mathrm{s}_{3}$ & $\mathbf{x}$ & $x$ & $\mathbf{x}$ & $\mathbf{x}$ & $s_{5}$ & $s_{1}$ & $\mathrm{~s}_{3}$ & $s_{7}$ \\
\hline$s_{4}$ & $s_{2}$ & $s_{6}$ & $\mathrm{~s}_{0}$ & $s_{4}$ & $x$ & $x$ & $x$ & \\
\hline$s_{5}$ & $x$ & $x$ & $\mathbf{x}$ & $x$ & $\mathrm{~s}_{3}$ & $s_{7}$ & $s_{1}$ & $s_{5}$ \\
\hline$s_{6}$ & $s_{6}$ & $s_{2}$ & $\mathbf{s}_{4}$ & $s_{0}$ & $x$ & $x$ & $x$ & $x$ \\
\hline$s_{7}$ & $x$ & $x$ & $x$ & $x$ & $s_{7}$ & $s_{3}$ & $\mathbf{s}_{1}$ & $\mathbf{s}_{s}$ \\
\hline
\end{tabular}

(b)
Fig. 3. (a) and (b) State transition diagram of 8-state Ungerboeck's TCM scheme ( $x$ denotes invalid transitions).

in the 4-state and 8-state cases differ. This is elaborated in Section III.

Simulation is used to verify that in both the 4-state and 8-state cases the 8-APSK TCM indeed outperforms the 8-PSK TCM as predicted by the analysis. Simulation results for Rayleigh, Rician, and shadowed Rician channels are presented in the paper.

Throughout, we assume that the effect of the fading on the phase of the received signal is fully compensated and that the fading amplitude is known, i.e., channel state information (CSI) is available. Also, we consider the case of ideal interleaving.

This paper is organized as follows. In Section II, we describe the system model and the various types of fading considered in this paper and describe an asymptotic error analysis for fading channels. In Section III, we discuss code design for asymmetric PSK signal sets, and in Section IV we present simulation results and give a reasoning for the observed results. Section V, contains a few concluding remarks.

\section{SYSTEM MODEL}

Fig. 4 shows the block diagram of the TCM scheme on a fading channel considered in this paper. Input bits are encoded by a rate $2 / 3$ convolutional encoder and mapped onto a 8 -PSK symbol set. Let

$$
\begin{gathered}
x=\left(x_{1}, x_{2}, \cdots, x_{i}, \cdots, x_{N}\right) \\
x_{i} \in\left\{s_{0}, s_{1}, s_{2}, s_{3}, s_{4}, s_{5}, s_{6}, s_{7}\right\}
\end{gathered}
$$

denote the symbol sequence before ideal interleaving. We assume that the receiver performs coherent detection and hence compensates the channel phase shift at the receiver. The channel produces a noisy discrete-time sequence. After deinterleaving this noisy discrete-time sequence, $\left\{y_{i}\right\}$ is written as

$$
y_{i}=\rho_{i} x_{i}+n_{i}
$$

Here, $n_{i}=\operatorname{Re}\left(n_{i}\right)+\operatorname{Im}\left(n_{i}\right)$ is a complex noise process. $\operatorname{Re}\left(n_{i}\right)$ and $\operatorname{Im}\left(n_{i}\right)$ are uncorrelated zero mean Gaussian r.v.'s each with variance $\sigma^{2}=N_{o} / 2 . \rho_{i}$ is a random variable representing the random amplitude of the received signal.

For a channel with only a diffuse multipath component the fading amplitude is Rayleigh distributed with a probability density function (pdf)

$$
P_{\rho}(\rho)=2 \rho e^{-\rho^{2}}, \quad \rho>0 .
$$

For a channel with a direct line-of-sight (LOS) component present, in addition to the diffuse multipath components, the fading amplitude is Ricean distributed with a pdf [2]

$$
\begin{gathered}
P_{\rho}(\rho)=2 \rho(1+K) e^{-K-(1+K) \rho} I_{0}(2 \rho \sqrt{(K(1+K)}) \\
\rho>0
\end{gathered}
$$

where

$K \quad$ ratio of the energies of the received signal in the direct and diffuse components;

$I_{0}(\cdot) \quad$ zero-order modified Bessel function of the first kind. Notice that $K=0$ for the case when the direct component has energy zero, which corresponds to the Rayleigh fading case. For large values of $K$ the channel exhibits slight fading. For $K \rightarrow \infty$ we have the AWGN channel. 


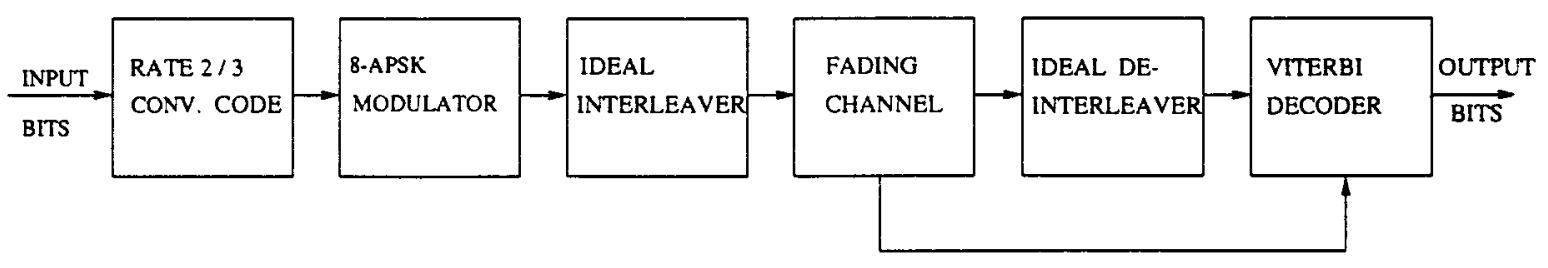

CHANNEL STATE INFO.

Fig. 4. System block diagram.

TABLE I

PARAMETERS FOR SHADOWED RICEAN FADING CHANNELS

\begin{tabular}{c|c|c|c}
\hline $\begin{array}{c}\text { Degree of } \\
\text { shadowing }\end{array}$ & $\sigma_{\rho}^{2}$ & $m_{\gamma}$ & $\sigma_{\gamma}$ \\
\hline Light & 0.1580 & 0.115 & 0.115 \\
Average & 0.1260 & -0.115 & 0.161 \\
Heavy & 0.0631 & -3.910 & 0.806 \\
\hline
\end{tabular}

When the direct LOS component is not constant but lognormally distributed, the fading amplitude models shadowed Rician fading. The fading amplitude may be expressed as

$$
\rho=\gamma+\eta_{0}+j \eta_{1}
$$

with

$$
\gamma=e^{\eta_{2}}+m_{\gamma}
$$

where $\eta_{0}, \eta_{1}, \eta_{2}$ are three independent Gaussian random variables with zero mean and variances $\sigma_{\rho}^{2}, \sigma_{\rho}^{2}$, and $\sigma_{\gamma}^{2}$, respectively. The pdf of the LOS component $\gamma$, which is a lognormally distributed random variable, may be expressed as

$$
P_{\Gamma}(\gamma)=\frac{1}{\sqrt{2 \pi} \sigma_{\gamma} \gamma} \exp \left(-\frac{\left(\log \gamma-m_{\gamma}\right)^{2}}{2 \sigma_{\gamma}^{2}}\right), \quad \gamma \geq 0
$$

Thus

$$
\begin{aligned}
P_{\rho}(\rho)= & \frac{\rho}{\sqrt{2 \pi} \sigma_{\rho}^{2} \sigma_{\gamma}} \int_{0}^{\infty} \frac{1}{\gamma} \\
& \cdot \exp \left(-\left[\frac{\left.\ln \gamma-m_{\gamma}\right)^{2}}{2 \sigma_{\gamma}^{2}}+\frac{\left(\rho^{2}+\gamma^{2}\right)}{2 \sigma_{\rho}^{2}}\right]\right) I_{0}\left(\frac{\rho_{\gamma}}{\sigma_{\rho}^{2}}\right) d \gamma
\end{aligned}
$$

The values for the $\sigma_{\rho}^{2}, \sigma_{\gamma}$, and $m_{\gamma}$ for light, average, and heavy shadowed Rician channels [11] based on the land-mobile satellite channel model of Loo [12] are given in Table I.

The pdf has been normalized in (1) and (2), that is the fading amplitude, $\rho$ has a mean-squared value of unity. This implies that the measured signal energy $E_{s}$ at the receiver represents the average signal energy per channel symbol. We assume that the fading amplitude, $\rho$, is perfectly estimated at the receiver. Ideal interleaving is assumed, that is, an interleaver with infinite depth is used. This assumption implies that the fading amplitudes are statistically independent and provides a memoryless channel model for the performance analysis.

The two sequences $\left\{y_{i}\right\}$ and $\left\{\rho_{i}\right\}$ are the inputs to the decoder which performs maximum likelihood (ML) decoding using Viterbi algorithm. An upper bound on the average error probability of this system is obtained as [3]

$$
P_{e}=\sum \sum_{\boldsymbol{x}, \hat{\boldsymbol{x}} \in \boldsymbol{C}} P(\boldsymbol{x}) \operatorname{Pr}(\hat{\boldsymbol{x}} / \boldsymbol{x})
$$

where $\boldsymbol{C}$ is the set of all valid sequences, $P(\boldsymbol{x})$ is the $a$ priori probability of transmitting $\boldsymbol{x}, \operatorname{Pr}(\hat{\boldsymbol{x}} / \boldsymbol{x})$ is the pairwise error probability, i.e., the probability that the decoder wrongly decodes to $\hat{\boldsymbol{x}}$ when $P(\boldsymbol{x})$ was the transmitted sequence.

\section{A. Asymptotic Performance Analysis}

The upper bound on the pairwise error probability for the system described above, i.e., coherent detection, perfect channel state information (CSI) and independent fading in each channel symbol, using a Rician model for the channel, is given as [3]

$$
\begin{aligned}
\operatorname{Pr}(\hat{\boldsymbol{x}} / \boldsymbol{x}) \leq & \prod_{i \in \eta} \frac{1+K}{1+K+\frac{E_{s}}{N_{0}}\left|x_{i}-\hat{x}_{i}\right|^{2}} \\
& \cdot \exp \left\{\frac{-K \frac{E_{s}}{4 N_{0}}\left|x_{i}-\hat{x}_{i}\right|^{2}}{1+K+\frac{E_{s}}{4 N_{0}}\left|x_{i}-\hat{x}_{i}\right|^{2}}\right\}
\end{aligned}
$$

where $\eta$ shows the set of all $i$ for which $x_{i} \neq \hat{x}_{i}$. For large SNR (7) simplifies to

$$
\operatorname{Pr}(\hat{\boldsymbol{x}} / \boldsymbol{x}) \leq \frac{\left((1+K) e^{-K}\right)^{l_{\eta}}}{\left(\frac{1}{4 N_{o}}\right)^{l_{\eta}} d_{p}^{2}\left(l_{\eta}\right)}
$$

where

$$
d_{p}^{2}\left(l_{\eta}\right)=\prod_{i \in \eta}\left|x_{i}-\hat{x}_{i}\right|
$$

Substituting (8) in (6) the average error probability for large SNR's may be approximated by [2]

$$
P_{e} \approx \alpha\left(L, d_{p}^{2}(L)\right) \frac{\left((1+K) e^{-K}\right)^{L}}{\left(\frac{1}{4 N_{o}}\right)^{L} d_{p}^{2}(L)}
$$

where

$L=\min \left(l_{\eta}\right)$ denotes the effective length of the code;

$d_{p}^{2}(L) \quad$ denotes the minimum-squared product distance between signal points of error event paths with effective length $L$; 
TABLE II

Product Distances FOR LENGTH 2 ERror EVENT FOR 4-STATE 8-PSK TCM

\begin{tabular}{c|c|c|c}
\hline $\begin{array}{c}\text { Normalized squared } \\
\text { product distances } \\
\text { of error events } \\
\text { for symmetric PSK }\end{array}$ & $\begin{array}{c}\text { Number of error } \\
\text { events with this } \\
\text { squared product } \\
\text { distance }\end{array}$ & \multicolumn{2}{|c}{$\begin{array}{c}\text { Normalized squared product distance } \\
\text { of the same error events after } \\
\text { introducing asymmetry of angle }\end{array}$} \\
\cline { 3 - 4 } & 16 & $\delta_{3}^{2}\left(\delta_{2, \theta}^{-}\right)^{2} / E_{s}^{2}=(4)\left(4 \sin ^{2}\left(\frac{3 \pi}{8}-\frac{\theta}{2}\right)\right)$ & $\theta=\pi / 12$ \\
\hline$\delta_{3}^{2} \delta_{2}^{2} / E_{s}^{2}=4(2+\sqrt{2}) \approx 13.656$ & 32 & $\delta_{1}^{2} \delta_{1}^{2} / E_{s}^{2}=4$ & 4 \\
\hline$\delta_{1}^{2} \delta_{1}^{2} / E_{s}^{2}=4$ & 32 & $\delta_{1}^{2}\left(\delta_{2, \theta}^{+}\right)^{2} / E_{s}^{2}=(4)\left(4 \sin ^{2}\left(\frac{3 \pi}{8}+\frac{\theta}{2}\right)\right)$ & 7.464 \\
\hline$\delta_{1}^{2} \delta_{2}^{2} / E_{s}^{2}=6.828$ & 16 & $\delta_{3}^{2}\left(\delta_{0, \theta}^{+}\right)^{2} / E_{s}^{2}=(4)\left(4 \sin ^{2}\left(\frac{\pi}{8}+\frac{\theta}{2}\right)\right)$ & 4 \\
\hline$\delta_{3}^{2} \delta_{0}^{2} / E_{s}^{2}=4(2-\sqrt{2}) \approx 2.344$ & &
\end{tabular}

$\alpha\left(L, d_{p}^{2}(L)\right)$ denotes the average number of code sequences having effective length $L$ and squared product distance $d_{p}^{2}(L)$.

For $K=0$, Rayleigh fading channel, the above approximation reduces to

$$
P_{e} \approx \frac{\alpha\left(L, d_{p}^{2}(L)\right)}{\left(\frac{1}{4 N_{o}}\right)^{L} d_{p}^{2}(L)}
$$

For $K=\infty$, AWGN channel, the approximation for $P_{e}$ is given by

$$
P_{e} \approx \alpha\left(d_{\text {free }}\right) Q\left(\frac{d_{\text {free }}}{2 \sqrt{N_{o}}}\right)
$$

where $d_{\text {free }}$ is the minimum-squared Euclidean distance of the code, $\alpha\left(d_{\text {free }}\right)$ denotes the multiciplicity of error events with distance $d_{\text {free }}$ and $Q(\cdot)$ is the Gaussian error-probability function. For large values of $K$ also (11) serves as a good approximation for the average error probability.

The upper bound on the pairwise error probability for a shadowed Rician channel in the above system is given as [2]

$$
\operatorname{Pr}(\hat{\boldsymbol{x}} / \boldsymbol{x}) \leq \prod_{i=1}^{N} \frac{1}{1+2 \sigma_{\rho}^{2} \frac{1}{4 N_{0}}\left|x_{i}-\hat{x}_{i}\right|^{2}} e^{-\left(C_{i} /\left(1+0.2 \sigma_{\gamma}^{2} C_{i}\right)\right)}
$$

where

$$
C_{i}=\frac{\frac{1}{4 N_{0}}\left|x_{i}-\hat{x}_{i}\right|^{2}}{1+2 \sigma_{\rho}^{2} \frac{1}{4 N_{0}}\left|x_{i}-\hat{x}_{i}\right|^{2}} e^{2 m_{\vartheta}}
$$

Substituting (12) in (6) the average error probability for large SNR's may be approximated by

$$
P_{e} \approx \frac{\alpha\left(L, d_{p}^{2}(L)\right)}{\left(2 \sigma_{\rho}^{2} \frac{1}{4 N_{o}}\right)^{L} d_{p}^{2}(L)} e^{-\left(D_{i} L /\left(1+0.2 \sigma_{\gamma}^{2} D_{i}\right)\right)}
$$

where

$$
D_{i}=\frac{e^{2 m_{\gamma}}}{2 \sigma_{\rho}^{2}}
$$

\section{CODE DESIGN}

Based on (9), (10), and (13), the code design criteria for Rician channels with small $K$, Rayleigh fading channels $(K=0)$ and shadowed Rician channels with perfect CSI are as follows.

1) Maximize the effective length of the code $L$.

2) Maximize the smallest product of the squared distances of signals along the error events of effective length $L$.

3) Minimize the multiplicity of error events, $\alpha\left(L, d_{p}^{2}(L)\right)$, at the smallest product distance.

Based on these code design criteria for fading channels we present new asymmetric PSK codes in this section.

Equations analogous to those in Section II-A can be obtained when no CSI is present at the receiver. The code design criteria for this case are also as above. For the AWGN channel and Rician channel with large $K$, based on (11), $d_{\text {free }}$ is to be maximized and $\alpha\left(d_{\text {free }}\right)$ is to be minimized.

\section{A. Design of 4-State Rate 2/3 8-APSK TCM}

Based on the code design criteria for fading channels 4-state rate $2 / 3$ symmetric 8 -PSK TCM schemes have been constructed in [2], [7], and [8]. Fig. 1(a) shows the symmetric 8-PSK signal set with relevant distance marked. Since parallel transitions limit the effective length to one, these should be avoided for applications in fading channels. Fig. 2(a) shows the state transitions of a fully-connected, 4-state TCM scheme without parallel transitions. The maximum effective length that a TCM scheme with one symbol per trellis branch can achieve is limited by the number of input bits and the number of encoder memories [2, ch. 5]. The maximum achievable effective length for a 4-state rate 2/3 8-PSK TCM scheme is two.Wilson and Leung [7] have suggested such a scheme that results in $L=2$. However, $d_{p}^{2}(2)$ has not been optimized resulting in a path with actual length 3 and effective length 2 with $d_{p}^{2}(2)=1.172 E_{s}^{2}$, where $\sqrt{E_{s}}=\delta_{3}$ is the radius of the circle in Fig. 1(a). In [8], Jamali and LeNgoc describe design rules which optimize $d_{p}^{2}(2)$ and improve the same to $2.344 E_{s}^{2}$. Fig. 2(c) shows the state transitions in matrix form of the 4-state rate 2/3 8-PSK TCM schemes obtained following these rules that achieve this minimum-squared product distance [8].

We show here that following the design rules of Jamali and LeNgoc for 8-PSK signal set with asymmetry as shown in Fig. 1(b) further increases the value of $d_{p}^{2}(2)$ [13]. The increase depends on the value of asymmetry $\theta$ (see Table II) and the maximum value achievable is four which is attained for an 
TABLE III

COMPARISON OF 4-STATE RATE 2/3 8-PSK SCHEMES

\begin{tabular}{c|c|c|c||c}
\hline & $\mathrm{U}[1]$ & $\mathrm{WL}[8]$ & $\mathrm{JN}[9]$ & $\mathrm{APSK}$ \\
\hline $\mathrm{L}$ & 1 & 2 & 2 & 2 \\
\hline$d_{p}^{2}(L)$ & $4 E_{s}$ & $1.172 E_{s}^{2}$ & $2.344 E_{s}^{2}$ & $4 E_{s}^{2}$ \\
\hline$d_{f r e e}^{2}$ & $4 E_{s}$ & $2.586 E_{s}$ & $3.172 E_{s}$ & $3.268 E_{s}$ \\
\hline
\end{tabular}

angle $\pi / 12$. Moreover, the free Euclidean distance also improves marginally compared to Jamali and Le-Ngoc. Table III summarizes the performance of known 4-state symmetric 8-PSK schemes with the last column showing the performance of the asymmetric 8-PSK scheme proposed here.

We first consider the Jamali and LeNgoc code obtained by the labeling shown in Fig. 2(c). In this code the minimum-squared product distance is achieved by the combination [2]

$$
\delta_{3}^{2} \delta_{0}^{2}=2.344 E_{s}^{2} \text {. }
$$

The diverging branches from a node have signal points separated by either $\delta_{1}$ or $\delta_{3}$. For actual length 2 (also effective length 2) error events if the squared distance between the diverging branches is $\delta_{3}^{2}$ then the squared distance between the merging branches is $\delta_{0}^{2}$ and if the diverging branch has squared distance $\delta_{1}^{2}$ then the merging branches have squared distance at least $\delta_{1}^{2}$. The pair of signal points contributing $\delta_{0}^{2}$ in (14) have to be on the merging branches. Observing columns of the matrix shown in Table II, it follows that the $\delta_{0}^{2}$ term in the above expression appears only in one of the following ways:

$$
s_{5} s_{6} \text { or } s_{1} s_{2}
$$

and in no other way, i.e., no other combination of the form $s_{i} s_{i+1}$ can appear as branch labels in the same level, since both $s_{i}$ and $s_{i+1}$ do not appear in any column in Fig. 2(c). Introduction of asymmetry as shown in Fig. 1(b) increases the distance among the pairs $\left(s_{5} s_{6}\right)$ and $\left(s_{1} s_{2}\right)$. This is the key to the improvement of the minimum-squared product distance. To elaborate, let the angle of asymmetry introduced be $\theta$ as shown in Fig. 1(b). Due to the asymmetry the signal points $s_{0}, s_{2}, s_{4}$, and $s_{6}$ do not change their position while other points change their positions. Let the new distances after introducing asymmetry be $\delta_{0, \theta}^{-}, \delta_{0, \theta}^{+}, \delta_{1}, \delta_{2, \theta}^{-}, \delta_{2, \theta}^{+}, \delta_{3}$ as shown in Fig. 1(b). Notice that the distances between error events of effective length and actual length 2 are given in the first column of Table II and the same squared product distances either decrease or increase after introducing asymmetry as shown in the third column.

Notice that the minimum-squared product distance $\delta_{3}^{2}\left(\delta_{0}^{+}\right)^{2}$ always increases; this is due to the fact that only the pairs $\left(s_{5} s_{6}\right)$ and $\left(s_{1} s_{2}\right)$ contribute to $\delta_{0}$ and asymmetry increases the distance among points of these two pairs. For an angle of asymmetry $\theta$, we have

$$
\delta_{3}^{2}\left(\delta_{0}^{+}\right)^{2}=(4)\left(4 \sin ^{2}\left(\frac{\pi}{8}+\frac{\theta}{2}\right)\right)
$$

which goes on increasing as $\theta$ increases. But the next higher squared product distance is $\delta_{1}^{2} \delta_{1}^{2}=4$. Hence, $\theta$ is increased up to that value for which

$$
4 \sin ^{2}\left(\frac{\pi}{8}+\frac{\theta}{2}\right)=1
$$

which gives $\theta=\pi / 12$.
TABLE IV

CODE PARAMETERS FOR 4-STATE 8-PSK TCM FOR SYMMETRIC AND ASYMMETRIC CASES

\begin{tabular}{c|c|cc|c}
\hline$\theta$ & $d_{p}^{2}(2) / E_{s}^{2}$ & \multicolumn{2}{|c|}{$d_{p}^{2}(3) / E_{s}^{3}$} & $d_{\text {free }}^{2} / E_{s}$ \\
\hline $0^{\circ}$ & 2.344 & $0.586 \times 0.586 \times 2$ & $=0.687$ & 3.172 \\
\hline $15^{\circ}$ & 4 & $0.268 \times 1 \times 2$ & $=0.536$ & 3.268 \\
\hline
\end{tabular}

The free Euclidean distance for the APSK TCM scheme introduced above is

$$
\left(\delta_{0}^{-}\right)^{2}+\left(\delta_{0}^{+}\right)^{2}+\delta_{1}^{2}=3.268
$$

which is marginally higher than for the Jamali and LeNgoc code (see Table III). It is therefore expected that this code will perform better over the AWGN channel also.

\section{B. Design of 8-State Rate 2/3 8-APSK TCM}

Fig. 3 shows the transitions of the 8-state Ungerboeck's TCM scheme [1] obtained by mapping by set partitioning in matrix form. This code, when combined with symmetric 8-PSK, has free Euclidean distance, $d_{\text {free }}^{2}=4.586 E_{s}$, an effective length, $L=2$, and minimum-squared product distance along error events of effective length $2, d_{p}^{2}(2)=8 E_{s}^{2}$. In fact, all the 64 length-two error events have the same squared product distance. In all cases, this squared product distance is achieved by the combination [refer to Fig. 1(a)]

$$
\delta_{3}^{2} \delta_{1}^{2} \text {. }
$$

In the term above, $\delta_{3}$ is contributed by diametrically opposite points and Fig. 1(a) shows the pairs, marked by similar shapes, that contribute to the $\delta_{1}$ in $d_{p}^{2}(2)$. This prompts us to introduce asymmetry by moving one point from the pairs that contribute to $\delta_{1}$ in such a way that $\delta_{1}$ increases while retaining diametrical oppositeness, thereby not changing $\delta_{3}$, as shown in Fig. 1(c). The $\delta_{3}$ distances contributed by diametrically opposite signal points remain the same. The new distances after introducing asymmetry are as shown in Fig. 1(c). Now, for the code based on the asymmetric signal set $d_{p}^{2}(2)$ is

$$
\delta_{3}^{2}\left(\delta_{1}^{+}\right)^{2}=(4)\left(4 \sin ^{2}\left(\frac{\pi}{4}+\frac{\theta}{2}\right)\right)
$$

which goes on increasing as $\theta$ increases. Hence, all product distances for length two error events go on increasing. Now, increasing $d_{p}^{2}(2)$ results in lower average bit error probability for asymptotically large SNR's.

The free Euclidean distance of the code is given by

$$
\left(\delta_{2}^{-}\right)^{2}+\left(\delta_{1}^{-}\right)^{2}+\delta_{1}^{2}
$$

which reduces with increasing asymmetry.

It is to be noted that the asymmetric 8-PSK signal sets for the 4-state and 8-state 8-APSK TCM schemes are different. In the 4-state case the asymmetric 8-PSK signal set is the superposition of a 4-PSK signal set with a rotated version of itself. However, in the 8-state case the asymmetric signal set is constructed by adding together the symmetrical 2-PSK signal set with three rotated versions of itself. The type of asymmetry introduced is dependent on the code itself and is introduced in such a way as to improve the minimum product distance of the code. 


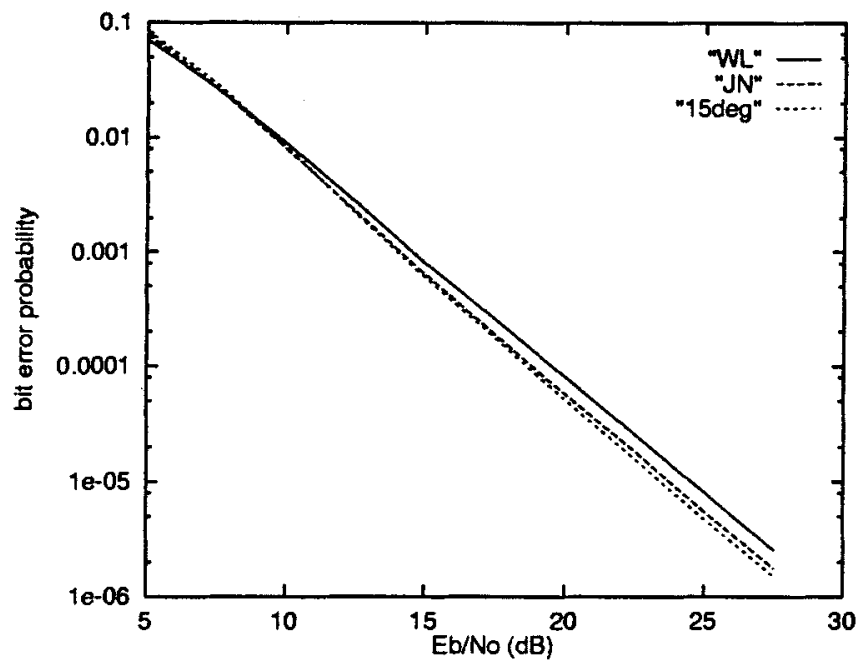

(a)

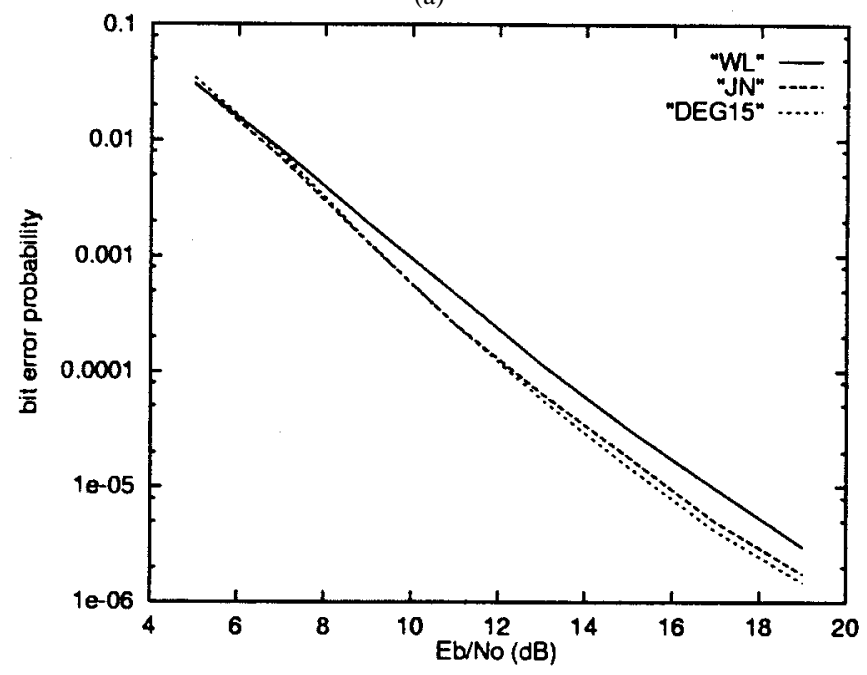

(c)

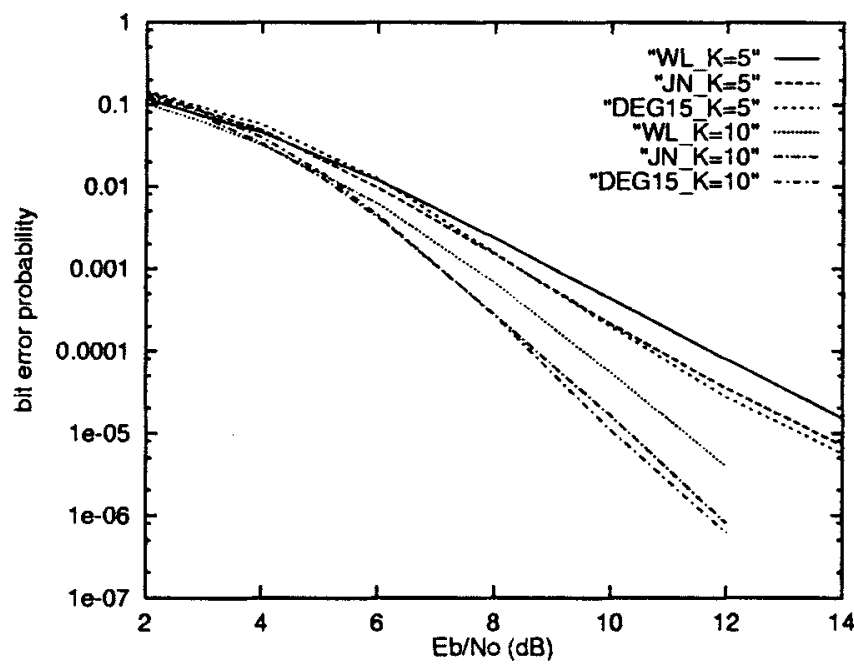

(b)

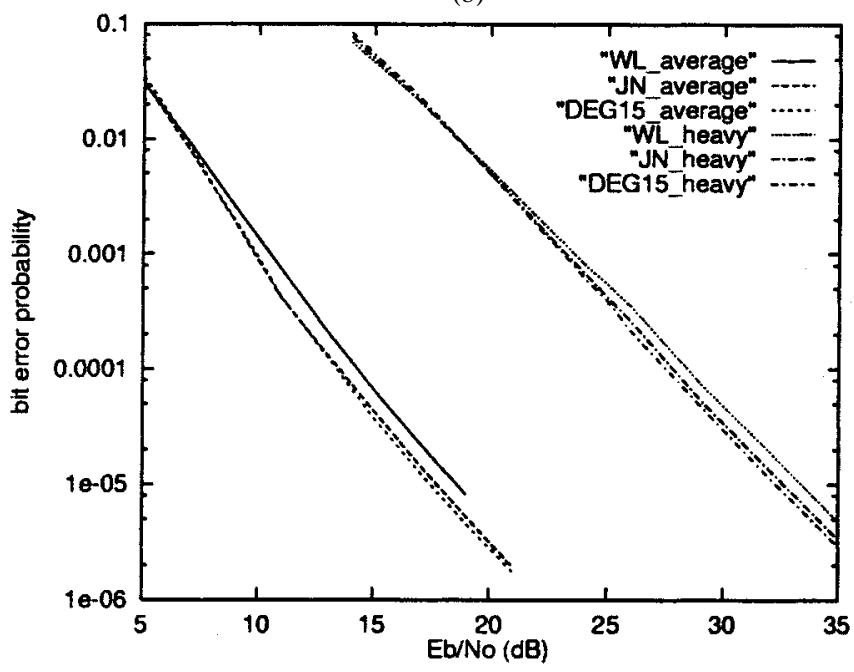

(d)

Fig. 5. Simulation results of the bit error rate performance for different 4-state 8-PSK codes over fading channels with perfect CSI. (a) Rayleigh, (b) Rician for $K=5$ and 10 , (c) light shadowed, and (d) average shadowed and heavy shadowed.

TABLE V

CODE PARAMETERS FOR 8-STATE 8-PSK TCM FOR ASYMMETRY ANGLE $<45^{\circ}$

\begin{tabular}{|c|c|c|c|}
\hline$\theta$ & $d_{p}^{2}(2) / E_{s}^{2}$ & $d_{p}^{2}(3) / E_{s}^{3}$ & $d_{\text {free }}^{2} / \bar{E}_{s}$ \\
\hline $0^{\circ}$ & 8 & $0.586 \times 2 \times 2=2.344$ & 4.586 \\
\hline $5^{\circ}$ & 9.389 & $0.586 \times 1.826 \times 1.826=1.953$ & 4.238 \\
\hline $10^{\circ}$ & $10 . \overline{07}$ & $0.586 \times 1.653 \times 1.653=1.601$ & 3.892 \\
\hline $15^{\circ}$ & 10.73 & $0.586 \times 1.48 \times 1.48=1.288$ & 3.546 \\
\hline $20^{\circ}$ & 10.73 & $0.586 \times 1.316 \times 1.316=1.015$ & 3.218 \\
\hline $25^{\circ}$ & 11.38 & $0.586 \times 1.155 \times 1.155=0.781$ & 2.896 \\
\hline $30^{\circ}$ & 12 & $0.586 \times 1 \times 1=0.586$ & 2.586 \\
\hline $35^{\circ}$ & 12.59 & $0.586 \times 0.852 \times 0.852=0.426$ & 2.290 \\
\hline $40^{\circ}$ & 13.14 & $0.586 \times 0.714 \times 0.714=0.299$ & 2.014 \\
\hline
\end{tabular}

\section{Simulation Results}

The APSK TCM codes discussed have been designed based on the fading channel code design criteria enumerated in Section III. Hence, the codes have been designed to have the max-
TABLE VI

CODE PARAMETERS FOR 8-STATE 8-PSK TCM FOR ASYMMETRY ANGLE $>45^{\circ}$

\begin{tabular}{c|c|c|c}
\hline$\theta$ & $d_{\mathrm{p}}^{2}(2) / E_{s}^{2}$ & $d_{p}^{2}(3) / E_{s}^{3}$ & $d_{\text {free }}^{2} / E_{s}$ \\
\hline $50^{\circ}$ & 14.128 & $0.362 \times 0.468 \times 0.468=0.0793$ & 1.298 \\
\hline $55^{\circ}$ & 14.553 & $0.187 \times 0.362 \times 0.362=0.0246$ & 0.911 \\
\hline $60^{\circ}$ & 14.928 & $0.068 \times 0.268 \times 0.268=0.0049$ & 0.604 \\
\hline
\end{tabular}

imum possible effective length 2 in the case of codes considered in this paper, and we have used asymmetry in the PSK signal set to maximize the minimum product distance along error events of length 2 . This leads to asymptotically good performance at large SNR's. For large SNR's, the error event probability is approximated by (9), (10), and (13) for the respective channels. At lower SNR, the minimum-squared product distances along error events of length 3 or more will dominate in the error event probability calculation if they are much smaller than $d_{p}^{2}(2)$. In such a situation, the error event probability can no longer be predicted by (9), (10), or (13). 


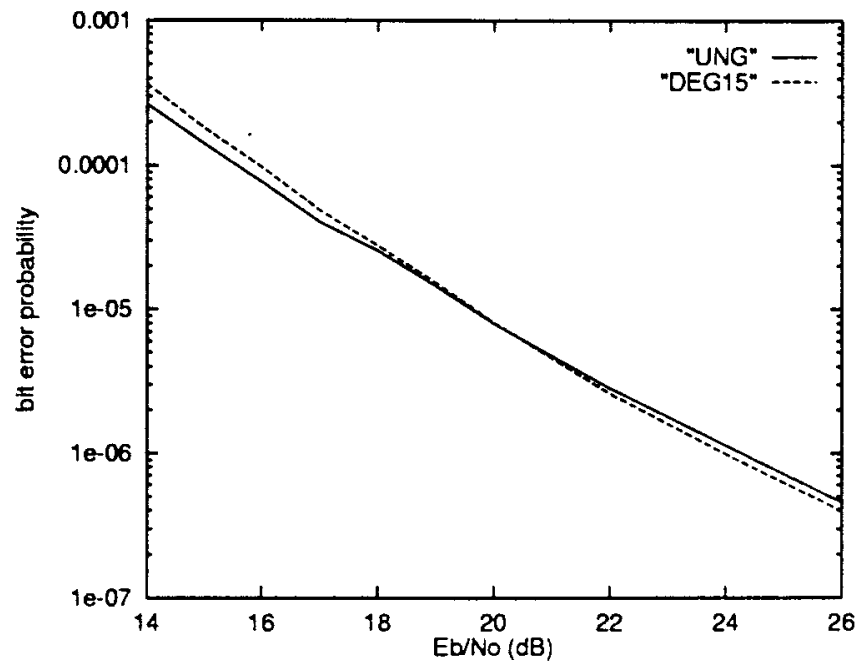

(a)

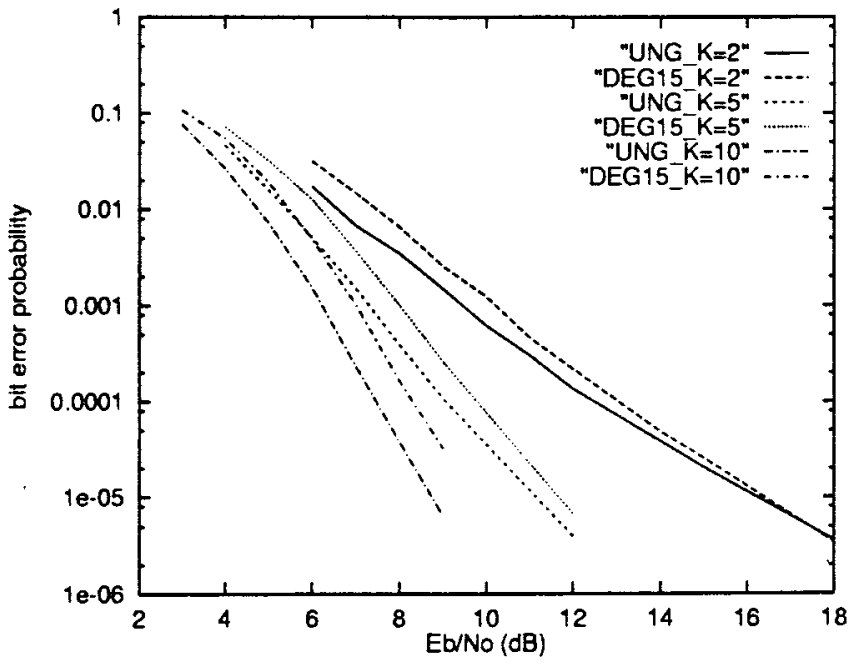

(b)

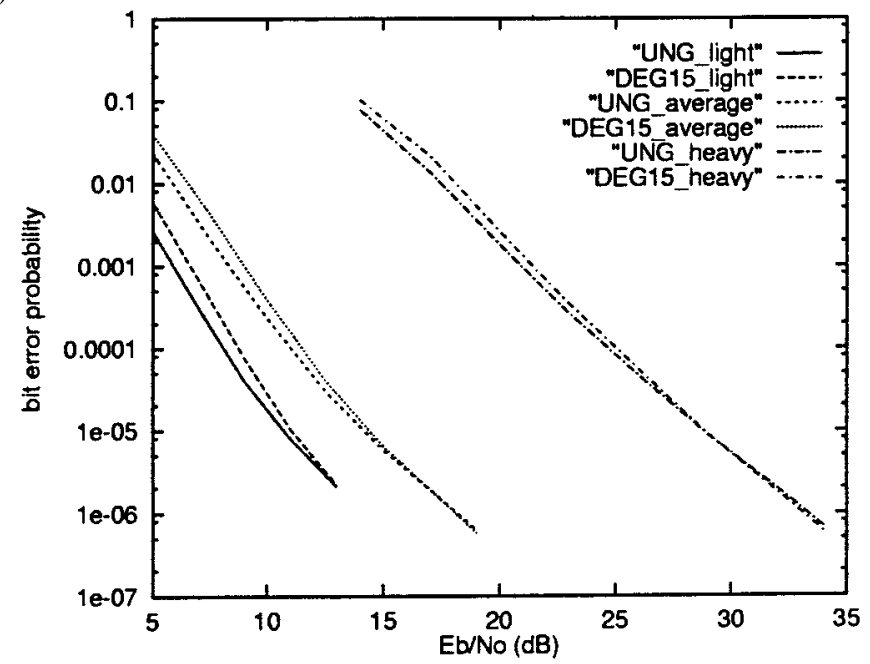

(c)

Fig. 6. Simulation results of the bit error rate performance for symmetric and asymmetric 8-state 8-PSK codes over fading channels with perfect CSI. (a) Rayleigh, (b) Rician for $K=2,5$, and 10, (c) light shadowed, and (d) average shadowed and heavy shadowed.

In the case of a Rayleigh fading channel, the contribution to the error event probability of the error events of length 3 is given by

$$
\frac{\alpha\left(3, d_{p}^{2}(3)\right)}{\left(\frac{E_{s}}{4 N_{o}}\right)^{3} \frac{d_{p}^{2}(3)}{E_{s}^{3}}}
$$

and the contribution to the error event probability of error events of effective length 2 is given by

$$
\frac{\alpha\left(2, d_{p}^{2}(2)\right)}{\left(\frac{E_{s}}{4 N_{o}}\right)^{2} \frac{d_{p}^{2}(2)}{E_{s}^{2}}}
$$

At lower SNR, (19) contributes more to the probability of error computation. For larger angles of asymmetry $(\theta)$, this quantity becomes smaller. This in turn implies that (20) becomes effective only at much larger SNR. Therefore, for larger angles of asymmetry, the APSK code performs better than the symmetric PSK code only at large values of SNR. Analogous arguments may be made for the Rician (small $K$ ) and shadowed Rician channels.

\section{A. Simulation Results for 4-State Rate 2/3 8-APSK TCM}

By listing out the length 2 and length 3 error events, it can be verified that for the symmetric 8-PSK TCM scheme $\alpha\left(2, d_{p}^{2}(2)\right)=16$ (see Table II) and $\alpha\left(3, d_{p}^{2}(3)\right)=64$. For the asymmetric 8-PSK $(\theta=\pi / 12)$ TCM scheme $\alpha\left(2, d_{p}^{2}(2)\right)=48$ (see Table II) if we increase the angle of asymmetry further this number comes down to 32 . The minimum length 3 product distance is achieved by the combination

$$
\left(\delta_{0}^{-}\right)^{2}\left(\delta_{0}^{+}\right)^{2} \delta_{1}^{2}
$$

For increasing $\theta$ this term becomes smaller and smaller and adversely affects the performance of the code at lower SNR. The different parameters are shown in Table IV for the symmetric 
and asymmetric cases. Interestingly, it is noted that $\left(\delta_{0}^{-}\right)^{2}\left(\delta_{0}^{+}\right)^{2}$ and $\delta_{1}^{2}$, the terms that contribute to the minimum product distance for length three error events, are the terms that contribute to the free Euclidean distance of the code (15).

Fig. 5 shows the results of the simulation carried out for Wilson and Leung code (WL), Jamali and LeNgoc code (JN) and the APSK code of this paper $\left(15^{\circ}\right)$. The decision depth of the Viterbi decoder was 18 symbols in both cases. The APSK code discussed in this paper shows improvement over the Jamali and Le-Ngoc code for $P_{e}$ smaller than $10^{-4}$ over all channels considered. There is an improvement of about $0.3-0.4$ $\mathrm{dB}$ around the bit error probability $10^{-5}$ over the Rayleigh fading channel and shadowed Rician channels. Over the Rician channels this improvement is about $0.2-0.3 \mathrm{~dB}$. An improvement in the range $0.2-0.4 \mathrm{~dB}$ implies $5-10 \%$ savings in power. The reason for the APSK code showing better performance over Rayleigh, Rician, and shadowed Rician channels is that it improves in both minimum product distance and free Euclidean distance over WL and JN codes (see Table III).

\section{B. Simulation Results for 8-State Rate 2/3 8-APSK TCM}

By listing the length 2 and length 3 error events it can be verified that $\alpha\left(2, d_{p}^{2}(2)\right)=64$ and $\alpha\left(3, d_{p}^{2}(3)\right)=128$ for $\theta<$ $45^{\circ}$. The minimum length 3 product distance for $\theta<45^{\circ}$ is achieved by the combination

$$
\delta_{0}^{2}\left(\delta_{1}^{-}\right)^{2}\left(\delta_{1}^{-}\right)^{2}
$$

and for $\theta>45^{\circ}$ by the combination

$$
\left(\delta_{2}^{-}\right)^{2}\left(\delta_{1}^{-}\right)^{2}\left(\delta_{1}^{-}\right)^{2}
$$

The various parameters are shown in Table $\mathrm{V}$ for angles of asymmetry less than $45^{\circ}$ and in Table VI for angles greater than $45^{\circ}$. An angle of asymmetry larger than $45^{\circ}$ corresponds to points $s_{1}, s_{6}, s_{2}, s_{5}$ crossing over the adjacent points $s_{0}, s_{7}, s_{3}, s_{4}$, respectively. At this point, a number of error event paths with length 3 , but squared product distance more than $d_{p}^{2}(3)$ begin taking values close to $d_{p}^{2}(3)$. Again, $\left(\delta_{2}^{-}\right)^{2}$ $\left(\delta_{1}^{-}\right)^{2}$ and $\delta_{1}^{2}$, the terms that contribute to the minimum product distance for length three error events, are the terms that contribute to the free Euclidean distance of the code (18).

Fig. 6 shows the results of the simulations carried out for the proposed 8-APSK signal set for $\theta=15^{\circ}$ (DEG15) and the symmetric 8-PSK case (UNG). The decision depth of the Viterbi decoder was 24 symbols in both cases. Fig. 6(a) shows that indeed the 8-APSK TCM code performs better than the symmetric TCM code over the Rayleigh fading channel at high SNR. At $P_{e}>10^{-6}$, there is an improvement of about $0.4 \mathrm{~dB}$ over the Rayleigh fading channel. Over the light, average and heavy shadowed Rician channels [Fig. 6(c)] the APSK code begins to perform better, albeit, at high SNR's. As introducing asymmetry has brought down the Euclidean distance of the resulting APSK code compared to the symmetric PSK code it is seen that over the Rician channels with large $K$ [Fig. 6(b)] the symmetric PSK code performs better than the APSK code. For Rician channels with smaller $K$ [Fig. 6(b)] the APSK code again crosses over at high SNR.

\section{CONCLUSIONS}

Asymmetric TCM schemes by increasing the minimum product distance at the shortest effective length result in gains without paying in bandwidth or power. In this paper, a 4-state and an 8-state 8-APSK TCM schemes have been presented that have the maximum effective length of two that is possible for such schemes and that achieves minimum product distances larger than the best known 8-PSK TCM schemes. Gains of the order of $0.4 \mathrm{~dB}$ over fading channels have been shown to be possible at data error probabilities. This implies about $10 \%$ savings in power over symmetric 8-PSK TCM. Many applications over fading channels involve battery supplied power and hence this saving is very meaningful for such applications. It must be remembered that symmetric 8-PSK TCM already achieves large gains compared to uncoded systems. Higher asymmetry angles may result in more gains but at much higher SNR's. However, bringing the points too close together makes a system more sensitive to phase jitter due to imperfect carrier synchronization and must be avoided. Also, the crossovers may begin to happen at impractically high SNR's.

\section{REFERENCES}

[1] G. Ungerboeck, "Channel coding with multilevel/phase signals," IEEE Trans. Inform. Theory, vol. IT-28, pp. 55-67, Jan. 1982.

[2] S. H. Jamali and T. LeNgoc, Coded-Modulation Techniques for Fading Channels. Norwell, MA: Kluwer, 1994, pp. 164-165.

[3] D. Divsalar and M. K. Simon, "The design of trellis coded MPSK for fading channels: Performance criteria," IEEE Trans. Commun., vol. 36, pp. 1004-1012, Sept. 1988.

[4] C. Schlegel and D. J. Costello, Jr., "Bandwidth efficient coding for fading channels: Code construction and performance analysis," IEEE J. Select. Areas Commun., vol. 7, pp. 1356-1368, Dec. 1989.

[5] E. Biglieri, D. Divsalar, P. J. McLane, and M. K. Simon, Introduction to Trellis-Coded Modulation with Applications. New York: MacMillan, 1991.

[6] D. Divsalar and M. K. Simon, "Trellis coded modulation for 4800-9600 bits/s transmission over a fading mobile satellite channel," IEEE J. Select. Areas Commun., vol. SAC-5, pp. 162-175, Feb. 1987.

[7] S. G. Wilson and Y. S. Leung, "Trellis-coded phase modulation on Rayleigh channels," in ICC'87 Conf. Rec., Seattle, WA, June 1987, pp. 21.3.1-21.3.5.

[8] S. H. Jamali and T. Le-Ngoc, "A new 4-state 8PSK TCM scheme for fast fading, shadowed mobile radio channels," IEEE Trans. Veh. Technol., vol. 40, pp. 216-222, Feb. 1991.

[9] J. Du and B. Vucetic, "New M-PSK trellis codes for fading channels," Electron. Lett., vol. 26, pp. 1267-1269, Aug. 1990.

[10] D. Divsalar, M. K. Simon, and J. H. Yuen, "Trellis coding with asymmetric modulations," IEEE Trans. Commun., vol. COM-35, pp. 130-141, Feb. 1987.

[11] P. J. McLane, P. H. Wittke, P. K.-M. Ho, and C. Loo, "PSK and DPSK trellis codes fast fading, shadowed mobile satellite communication channels," IEEE Trans. Commun., vol. 36, pp. 1242-1246, Nov. 1988.

[12] C. Loo, "A statistical model for a land mobile satellite link," IEEE Trans. Veh. Technol., vol. VT-34, pp. 122-127, Aug. 1985.

[13] L. V. Subramaniam, B. S. Rajan, and R. Bahl, "A 4-state asymmetric 8-PSK TCM scheme for Rayleigh fading channels," in Proc. 1998 Int. Symp. Information Theory (ISIT'98), Cambridge, MA, Aug. 16-21, 1998, p. 252 


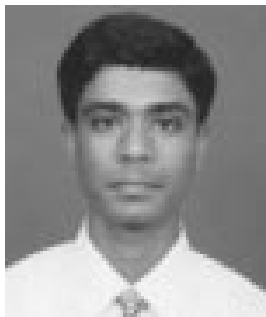

L. Venkata Subramaniam (S'93-M'98) was born in New Delhi, India. He received the B.E. degree in electronics and communications engineering from the P.E.S. College of Engineering, University of Mysore, Mysore, India, the M.S. degree in electrical engineering from Washington University, St. Louis, MO, and the Ph.D. degree in electronics from the Indian Institute of Technology, New Delhi, India, in 1991, 1993, and 1998, respectively. His dissertation focused on the areas of coding and channel modeling.

From 1993 to 1996, he was involved with the project Advanced Object Visualization Techniques (ADOVIT) for underwater scenario sponsored by the Department of Electronics. He is presently a Research Staff Member with the IBM Solutions Research Centre, New Delhi, and is working on speech recognition and audio-driven facial animation.

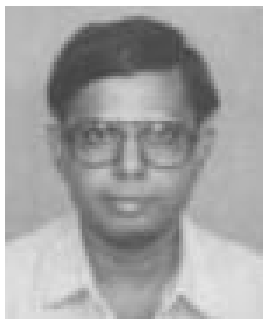

B. Sundar Rajan (S'84-M'91-SM'99) was born in the Tiruchirappali District, Tamil Nadu, India. He received the B.Sc. degree in mathematics from Madras University, Madras, India, the B.Tech. degree from the Madras Institute of Technology, Madras, and the M.Tech. and Ph.D. degrees from the Indian Institute of Technology, Kanpur, India, in 1979, 1982, 1984, and 1989 , respectively.

After serving as a Faculty Member for a semester in the Department of Electronics and Computer Science at Roorkee University in 1990, he became a Faculty Member with the Department of Electrical Engineering at the Indian Institute of Technology, Delhi, from 1990 to 1997. Presently, he is an Associate Professor in the Electrical Communication Engineering Department of the Indian Institute of Science, Bangalore. His primary research interests are in error-correcting codes, coded modulation, and cryptology.

Dr. Rajan is a Senior Member of the IEEE Information Theory Society and Communications Society and a member of the American Mathematical Society

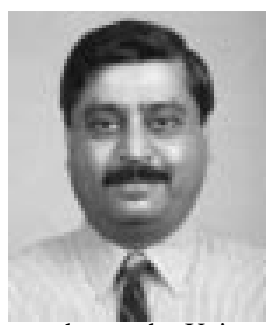

Rajendar Bahl (M'95) is a Professor at the Centre for Applied Research in Electronics (CARE), Indian Institute of Technology, Delhi, India. He has been working in the area of underwater electronics for over 20 years and currently heads the Signal Processing Group at CARE. The group offers specialized graduate courses in underwater systems and signal processing. His research interests include 3-D imaging sonars for undersea robotic applications and design of compact DSP hardware. He was a Visiting Researcher at the University of Technology, Loughborough, U.K., during 1981 and 1986 and a Senior Research Associate at the Naval Postgraduate School, Monterey, CA, during 1989-1990.

Dr. Bahl is Fellow of the Institute of Electronics and Telecommunications Engineers (India). 